\title{
A Theoretical Model Applying Fuzzy Logic Theory for Evaluating Personnel in Project Management
}

\author{
Darko Galinec \\ Ministry of Defence, Republic of Croatia \\ Slavko Vidović \\ University of Zagreb
}

\begin{abstract}
Project team work depends considerably on the contribution of its members and its management as well. Cooperation among members depends on the leader who is supposed to encourage interactions, growth and knowledge exchange among members. To identify the importance of each individual, a leader/manager must evaluate individual contributions. On the basis of such assessments, necessary and possible actions will be undertaken to improve the achievements of the team as a whole. A leader usually has defective and incomplete information at his disposal and, besides, is subjective. To solve the aforesaid problem, fuzzy approach and fuzzy logic are used. Fuzzy logic, as a method for soft-computing, as input values employs data with the following features; uncertainty and partial verity, indistinctive borders among particular categories. Fuzzy evaluation system has been designed to reduce evaluation subjectivity.
\end{abstract}

\section{Classical and Fuzzy Approach to Classification}

Classical theory of sets has considerably contributed to the development of numerous scientific program solutions designed for decision making where phenomena can be precisely measured. In problem fields where there are no such measuring possibilities, classical set theory does not render good results. Employment of classical sets theory can lead to misinterpretation of data and knowledge. Sometimes transfer from one set to another is not precise but gradual. In such cases, to classify members into well defined groups, it is necessary to define arbitrary boundaries among sets. The problem is even bigger when information needed for decision making is not available in exact, mathematic form, so judgement must be made with certain percentage of uncertainty. Such reflections have resulted in the development of fuzzy sets theory, which according to Zadeh (Schneider, Kandel, Langholz \& Chew, 1996) differs from classical theory in one key standpoint: An element can belong to the fuzzy set, be completely excluded from the fuzzy set, or it can belong to the fuzzy set to any intermediate degree between these two extremes. Membership or not to a fuzzy set and its presentation through certain values makes classical theory a special case of fuzzy set theory. In classical sets theory a member can belong to a set if, and only if, it fulfils all conditions of the membership, otherwise it doesn't belong to the set. If, for example, the set «satisfying" contains the values from 8 to 14, an individual to get such a mark must have at least 8 and at the most 14 points. If he/she has 7.9 points, he/she will not belong to the set "satisfying». Since there are no indicators showing how close an eliminated individual is to a set membership, an individual having only 1 point will be eliminated from the set "satisfying» (and ranged, put into the set «unsatisfying») as well as an individual having 
7.9 points. Classifying the one having only 1 point and the one having 7.9 points into the same category, i. e. ranging the persons having 7.9 and 8 points into different categories leads to, if we apply classical sets theory, inconsistency.

\section{Necessity to Evaluate Performance of the Members within Program}

Organizational, financial, human and material resources are requisite for the information system development. According to this paper, human resources are in the centre of a star, other resources being points around it. Professional profile of the necessary staff belongs mainly to informatics field, minor part relating to the clerical staff. A program manager, the one who is the most responsible for the plan realization, must have possibility to evaluate to the utmost the contribution and reliability of each member, so that each member could be managed in a way that leads to success - successful completion of the program. In this way behavior and matter of fact contribution of each individual will be determined, as well as favorable moment and the ways suitable for the application of available mechanisms for human resources management. Because of impossibility to evaluate contribution of each individual (which is immanent to evaluation process), i.e. inability to define and determine precise and strict bounds between possible evaluation ratings for an individual, with a view to find the adequate way to solve the problem, ratings in this work are represented as fuzzy sets. Their mutual bounds are not strictly defined, changes are gradual, common part being maintained. Due to complexity, numerous problem situations can not be solved in a distinctive way. To resolve real problems, like program management of human resources, financial, and organizational resources, as well as the tracking of their development, we lack timely and reliable data. Reasons are numerous and can be found inside (inadequate competency and to small a staff, interpersonal relations, motivation, decision making in connection with priorities and necessary financial support, planning and acquisition of necessary equipment) and outside (political decisions, economic measures, economic stability, international situation etc.). If, in a better case, there are data, they are late, incomplete, indefinite, not precise, in short, doubtful and because of that considerably subject to subjective assessments of an individual or a group resolving the problem. In spite of the aforesaid, it is necessary to create the best possible basis for rational decision making and problem elaboration, calculate the parameters value and create solution rules. It is not possible to eliminate completely assessment subjectivity, but it is possible to shape it and thus reduce its influence on decision making and management.

\section{Performance of the Members in Programs and Projects}

To obtain synergetic staff efficiency and rise in proficiency it is necessary, apart from material and financial resources, to provide for the other prerequisites. They are reflected in providing pleasant working environment, possibility to discuss assigned duties, expressing opinion about problems in good faith, respecting individual opinions, views and ideas, solution of possible conflicts on the basis of analysis and arguments, getting familiar with assigned duties and unambiguous assignment to a particular team member. Problems in connection with duties and related risks should be foreseen and actions should be undertaken to prevent problem occurrence. If such actions are not possible, problems must be solved without delay to minimize bad consequences. 


\section{Program Management}

Program management involves planning, organizing and control of a system development. Program management relates more to the prediction of possible events and less to the analysis of the present facts. It represents coordination of the tasks assigned to the team members, its basic duty being restraint of changes. Efficient leadership is, among other things, the result of actions taken by the leader and led team members and actual work situation. Very often such a situation is not simple - it depends on the number and complexity of the influencing factors. It is very difficult, sometimes even impossible, to overcome them all. So, it is necessary to identify the most important ones, which in a particular case have the biggest influence.

As for the program manager, amongst all factors, the most appreciated factor is the staff. The staff should be managed in a way which will provide for the best results and insure success of the work. In this connection he/she must be able to accelerate the accomplishment of the assigned tasks, on the assumption that there is no one and the same way to manage and lead a team, a way which will be suitable for all problem situations. Because of the aforementioned restrictions - subjectivity which is immanent quality of a human being, leader can develop fuzzy evaluation models in order to minimize subjectivity and to make his assessment objective as much as possible, using imprecise and uncertain values - the only ones at his disposal. A possible evaluation model is represented in this work.

\section{Fuzzy Approach to Problem Solving}

The goal for a role-oriented organization is to move away from traditional notions of how organizations must be structured, while accommodating both data-centric, traditional hierarchies in coexistence with newer, innovative structures. It is not important whether roles are insourced or outsourced. Identifying and staffing individual functions is the consequence of determining whether the roles can be best filled by internal or external resources, and whether it is appropriate to supplement existing personnel with part-time contractors or systems integrators. A role orientation is not a function, it breaks up requirements into a grouping of capabilities that can be fulfilled in several ways, including teams, specialty centers and by individuals assuming more than one function. The emphasis is on communications, process and governance. Each is critical in supporting the flexibility to change as requirements dictate.

\section{Fuzzy Logic Theory}

The membership function $\mu$ associated with a fuzzy set assigns degrees of membership to elements in the set. The extent to which an element belongs to a given fuzzy set is called the grade of membership or degree of membership. For example, we can assign the following values to the membership function associated with the temperature of the beer (Schneider, Kandel, Langholz \& Chew, 1996, p. 25): the degree of membership of $38^{\circ} \mathrm{F}$ beer in the fuzzy set of cold beers is $1, \mu(38)=1$, but the degree of membership of $46^{\circ} \mathrm{F}$ beer is only $0.2, \mu(46)=0.2$. The use of fuzzy set theory is very appealing in 
cases where the decision must be made in environments in which the information available is incomplete, vague, or ambiguous. The result, referred to as the certainty factor (CF), which is computed for each decision process, represents the degree of belief that the decision reached is correct. Note that although the certainty factor can have a value in any range, it is commonly normalized to the interval from 0 to 1 . Since the creation of logic rule that every statement is at the same time true and false, there have been some doubts about that (Bojadziev \& Bojadziev, 1997, p. 41). Although the roots of such reflections can be found in Aristotel, Bool, Peirce, Vasiliev, in contemporary sense we must consider Lukasiewicz's and Post's early works. Independently from each other, these authors have systematically defined multi-valued logic systems, Lukasiewicz being motivated by philosophical reasons and Post by mathematic ones. Lukasiewiscz in his work «On determinism» states if allegations about future events are true (sign 1) or false (sign 0 ), then the future is as much determined as the past, the only difference is that the future has not happened yet (Gabbay \& Guenthner, 1994, p. 72). The ancient Epicurus teaching supported indeterminism and rejected the principle of logic bivalence, while the Stoics accepted it and strongly advocated determinism (Gottwald, 1996, p. 8). To overcome the dead-end of determinism, Lukasiewicz abandoned the traditional rule on the elimination of the third one, which reads: «every allegation is whether true or false in the same sense, i.e. every allegation must be assigned one and only one value - truth or untruth». He added the third value - possible (sign $1 / 2$ ) and thus introduced the principle of logic threevalency. Later on he widened the set of possible values permitting number of values $n \geq$ 3 from $[0,1]$, introducing $n$-valued or multi-valued logic by means of infinite and countable set of rational numbers $Q$. If truth values are represented by all real numbers in $[0,1]$, i.e. truth set is $T_{\infty}=[0,1]$ (the set of real numbers is infinite and uncountable) then multi-valued logic is logic where judgements can assume infinite number of values (infinite valued logic) or standard Lukasiewicz logic. There is correspondence between fuzzy sets and infinite valued logic. Complement, intersection and union of fuzzy sets correspond respectively to negation, conjunction and disjunction of infinite valued logic. Fuzzy logic uses fuzzy sets theory as a main tool, basic mathematic ideas of fuzzy logic originated from infinite valued logic, and that is the connection between these two logics. Fuzzy logic can be considered an extension of infinite valued logic since it incorporates fuzzy sets and fuzzy relations (Bojadziev \& Bojadziev, 1997, p. 43).

The triangular membership function, see equation (1) and Figure 2 (Appendix A) is the most frequently used function and the most practical, but other shapes are also used (McNeill, Thro \& Thro, 1994, p. 33). It is very often used in fuzzy applications for fuzzy controller, managerial decision making, business and finance, social sciences, etc. It can be created in a simple way, on the basis of small amount of information (Bojadziev \& Bojadziev, 1997, pp. 22-23). 


\section{Methodological Steps for Model Construction}

The evaluation system is made by defining membership functions of linguistic variables, through the research in which author has shaped and implemented the original fuzzy evaluation system with that kind of internal structure - with original linguistic variables and original business rules - which enables tracking of the level of efficiency of the members of the project teams within the program, using incomplete (the only available) input values. It is the result of the execution of the following methodological steps:

1. The choice of the membership function of form: triangular

2. The choice of the number of fuzzy linguistic variable sets: 4

3. The determination of the width of the membership function interval: $[1,7]$

4. The construction of fuzzy production rules.

A system like this is meant to increase the level of efficiency of leadership and control, in all project/program phases, by performing ex-post evaluation of the work contribution of each member.

Membership functions of the linguistic variables were created, as were the linguistic variables themselves, based on the results the author produced by research conducted while leading complex informatics projects within programs. In order to successfully lead team members and control of the proceedings of the whole program, in the author's opinion, it was necessary to create a system with such a form and width of membership functions of linguistic variables as to enable the necessary evaluation of the project and program team members' work. These are the membership functions used to create the input and output values of the system, in the creation of which the existing working conditions and effectively available information decision making basis were taken into consideration. It consists of incomplete, subjective information which is to be used in the best manner possible, since no other is available. The triangular membership function has been chosen for reasons stated in the previous part of the paper (McNeill, Thro \& Thro, 1994, p. 33; Bojadziev \& Bojadziev, 1997, pp. 22-23). In its definition the author took into account that the evaluation interval should neither be too narrow nor too wide. If it were too narrow, with a small number of possible membership function values (two, for instance), the evaluation criteria would need to be very high, that is, it should be possible to speculate that this is an efficiency evaluation of a team with a high level of excellence. This assumption is hard to accept, because not only the most excellent individuals take part in programs for a certain type of work. If we would, in contrast, use too wide an interval, it would be difficult for the evaluator to conduct the evaluation, since it is hard for a human to decide on a specific value when the granulation level is too high. Therefore each of the membership functions of linguistic variables has a predetermined evaluation interval $[1,7]$. The number of membership functions for each linguistic variable is 4 and this is not only within previously stated demands, but it also enables the number of fuzzy rules to be neither too big, nor too small (in reality, it equals 16 for calculating each output linguistic variable). 


\section{Fuzzy Evaluation Model}

Since different kinds of uncertainty can be well explored and described within theory of fuzzy sets, instead of insisting upon precise and sharp bounds, necessary linguistic expressions are formalized by fuzzy sets with overlapping possibilities.

According to Schneider, Kandel, Langholz \& Chew (1996) in «Outline of a New Approach to the Analysis of Complex Systems and Decision Processes» Zadeh states in his principle of incompatibility: As the complexity of system increases, our ability to make precise and yet significant statement about its behavior diminishes until a threshold is reached beyond which precision and significance (or relevance) become almost mutually exclusive characteristics.

\section{Linguistic Variable and Membership Function}

As for fuzzy logic, expression used in natural language to describe some phenomena having fuzzy value is called a linguistic variable. A linguistic variable is often described in terms of its fuzzy space. This space is generally composed of multiple, overlapping fuzzy sets, each fuzzy set describing a semantic partition of the variable's allowable problem state (Cox,1994, p. 40). For example, the linguistic variable "Responsibility» is broken down into four fuzzy sets: "unsatisfying» (UN), «satisfying» (SA), «successful» (SU) and "very successful» (VS). This total problem space, from the smallest to the largest allowable value, is called the universe of discourse. The universe of discourse for the linguistic variable "Responsibility» is 1 to 16 measurement units - points in our example. Linguistic variable «Ability», «Reliability», and "Resulting value» can be modeled by means of sets $A B I, R E L, R E V$ containing certain number of values, see expression (6) (Appendix A).

\section{Fuzzy Rules and Logic Operations}

Preconditions and conclusions about phenomena can be found within fuzzy rules which create the knowledge basis on a certain phenomenon. Knowledge is presented as the following rule: «If $\mathrm{P}$ then $\mathrm{C}$ », $\mathrm{P}$ represents rule precondition and $\mathrm{C}$ its conclusion. Rule precondition consists of clauses mutually connected with logic operators. Conclusion consists of truth statement. Creation of fuzzy rules basis presents the most complex part of the work since collected knowledge about systems behavior is presented in a formal shape, thus containing the elements of artificial intelligence.

Membership function which refers to a fuzzy set joins degree of membership to set members. Membership to a certain fuzzy set is described as a degree of membership or grade of membership and refers to precondition on phenomenon. Certainty factor, CF, which refers to the conclusion on phenomenon is calculated for each decision making process and represents degree of belief that decision is correct. Although certainty factor can use the values from any interval, it is usually normalized on the interval $[0,1]$.

True value reaching procedure by means of fuzzy reasoning includes implicitly elaboration of all activated rules together. Each of the aforesaid rules contributes to the conclusion in a certain degree, that degree being equal to the degree to which is 
satisfied the portion of the rule referring to the precondition. Such an imitation process resembles to human deduction - a man making decisions takes into account different facts, each of them having particular influence on the final decision. The choice of appropriate logic operator represents an important part of a fuzzy system design. Originally, theory of fuzzy sets formulates standard Zadeh's minimum, maximum and complement operations.

Since 1965 a few classes of logic operators satisfying corresponding axioms have been introduced for each of the aforementioned operations (Rudas, 1999, p. 1).

By acceptance of some basic conditions, by means of t-operators, a huge class of operation sets for union and section has been created. T-norm concept and t-co-norm has been originally developed by Menger in 1942 within theory of probabilistic metric spaces. Since then, numerous types of t-operators have been developed. In many applications, such common operators are not efficient, so there are demands for compensational behavior (Rudas, 1999). Because of the aforesaid, compensation operators are introduced.

\section{Evaluation Model Structure}

Figure 1 displays input and output originally developed linguistic variables of a fuzzy system model.

Figure 1. The Structure of Modules

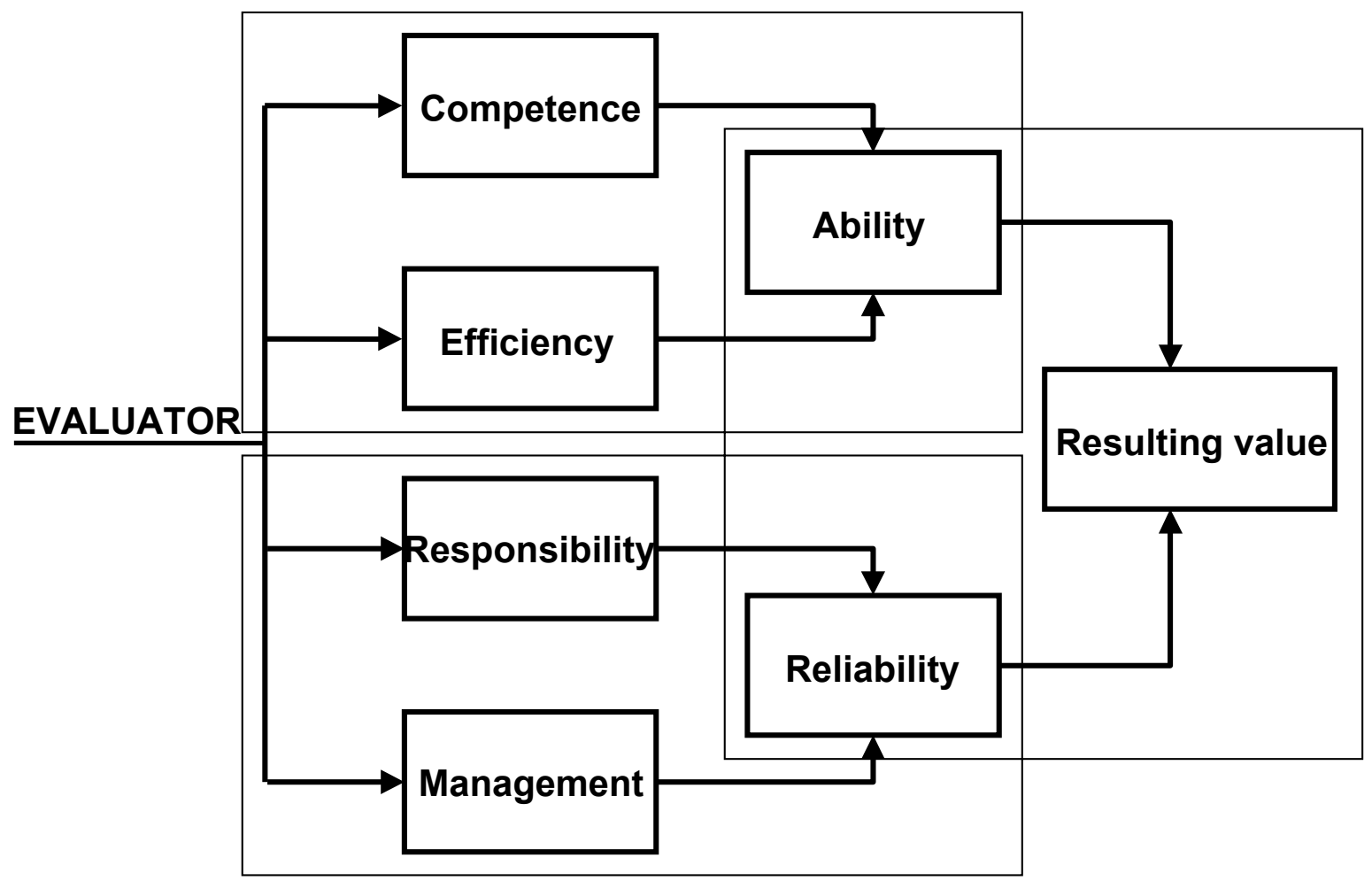

The first input module contains the input linguistic variable «Competence» which refers to the level of knowledge and expertise in the problem area referring to the performance 
of the members of the program, and is a result of the formal education of the team members, including completed courses and training. "Efficiency» represents the rate of produced effect of an individual measured by the volume and quality of the execution of the assigned tasks. Both dimensions of the program member affect the level of his «Ability», which is a linguistic variable that represents the first output value of the model. The other linguistic variable input module includes «Responsibility» as a measure of gravity and excellence of task execution performance, and «Management» that acknowledges the fact that within the modern organization forms nowadays the prevailing opinion is that all members of the team are management oriented managers, regardless whether they have subordinates or not. If the latter is the case, they are managers of their own tasks only. The output linguistic variable that is created within this module is «Reliability», and as a result of the two input variables it represents the measure of certainty with which the program manager can rely on the person in question, in order to maintain the quality and the dynamics of the work. The third module uses output values of the previous two modules as its input values, and its goal is to put together the level of quality of «Ability» and "Reliability» of a specific individual, so that it could, based on the derived «Resulting value», finally be decided on his or her future, form and type of cooperation in the common work.

\section{Conclusions}

A complex work like program and project elaboration and management, among other things, strongly requires qualitative evaluation of human capabilities and capacities in order to manage them. Improvisation and subjective evaluation should be reduced as much as possible. The aforesaid can be achieved by the development and employment of models which will produce decisions made on the basis of more objective assessments. By means of fuzzy approach and fuzzy logic employment, the system model supporting evaluation processes and decision making is rendered possible. Fuzzy evaluation model is complex and structured. According to its modules, fuzzy evaluation of individual characteristics is made, in conformity with beforehand defined rules modeling relationship among characteristics. On the basis of calculated output module values, the resulting, final evaluation value is reached. Shaped model is adaptable, it can be improved according to the business needs, introducing changes through the selection of features - linguistic variables, their values, and interaction rules as well - until satisfactory shape is achieved. 


\section{Appendix A. Formulas, Graphs and Comments \\ Membership Functions}

$\mu_{\text {triangle }}(x)=\left\{\begin{array}{c}0, x<a_{1} \\ \frac{x-a_{1}}{a_{M}-a_{1}}, a_{1} \leq x \leq a_{M} \\ \frac{x-a_{2}}{a_{M}-a_{2}}, a_{M} \leq x \leq a_{2} \\ 0, x>a_{2}\end{array}\right.$

Figure 2. The Triangular Membership Function

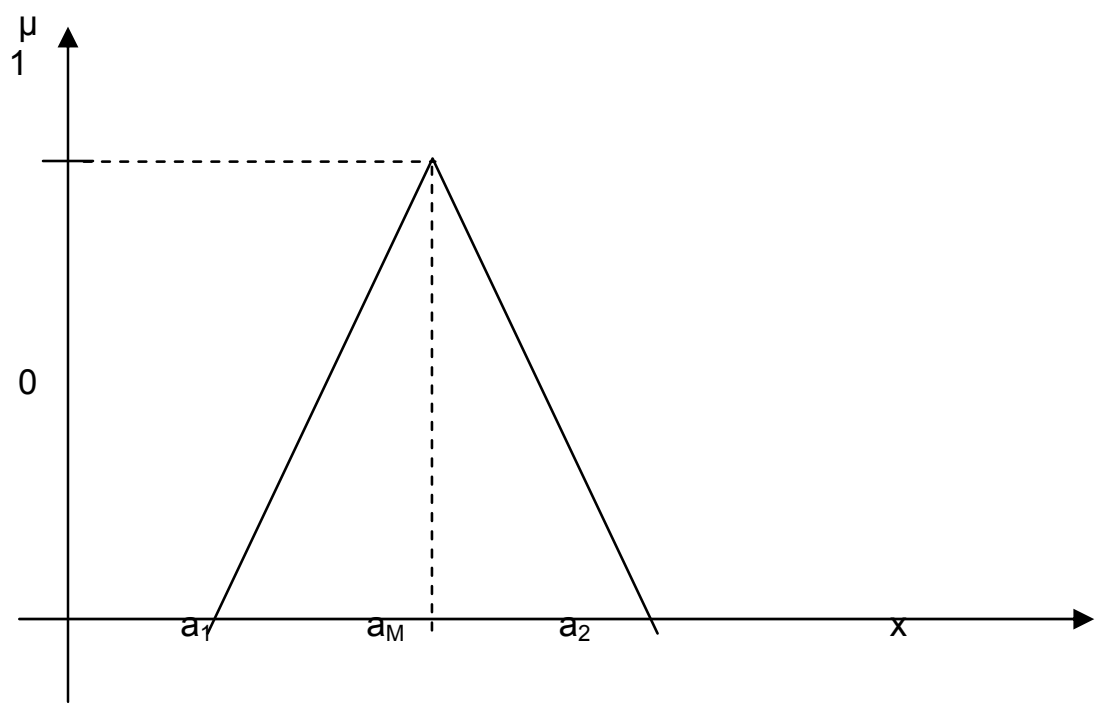

Other shapes are also used (see equation 2), as shown in figure 3. One is the trapezoid which contains more information than triangle.

$\mu_{\text {trapezoid }}(x)=\left\{\begin{array}{l}\frac{x-a_{1}}{b_{1}-a_{1}}, a_{1} \leq x \leq b_{1} \\ 1, b_{1} \leq x \leq b_{2} \\ \frac{x-a_{2}}{b_{2}-a_{2}}, b_{2} \leq x \leq a_{2} \\ 0, x<a_{1} \text { ili } x>a_{2}\end{array}\right.$ 
Figure 3. The Trapezoidal Membership Function

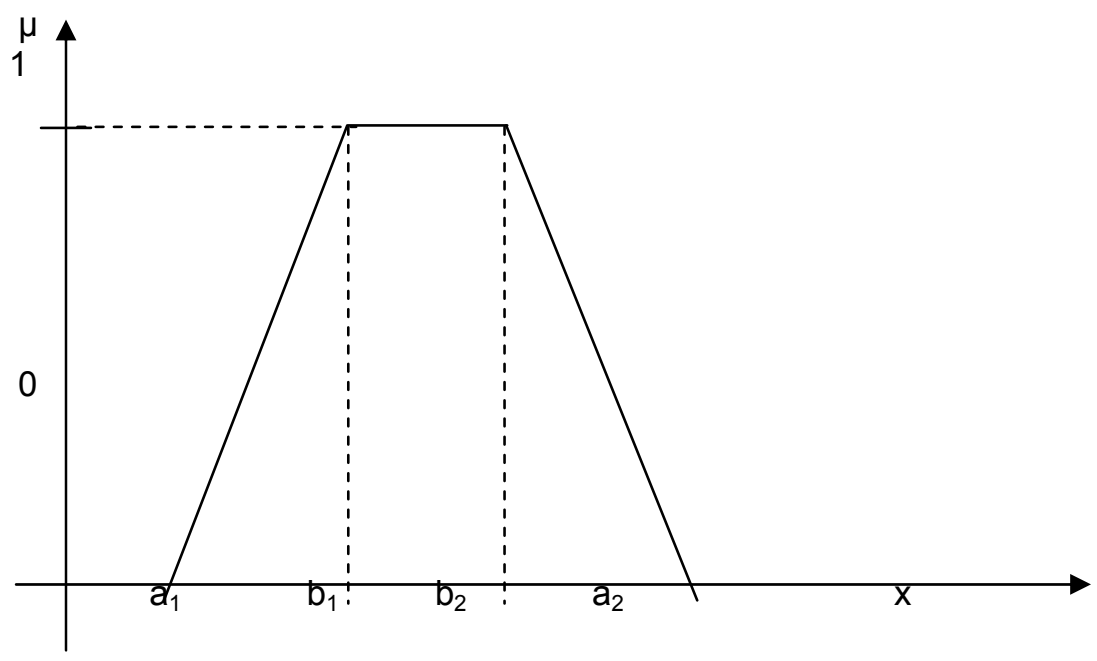

A fuzzy set can also be represented by a quadratic equation which produces a continuous curve. Three shapes are possible: $S, \pi$ (bell) and $Z(1-S)$ given by equations (3), (4) and (5). Functions are shown in figure 4.

$\mu_{S}(x)=\left\{\begin{array}{l}0, x \leq a_{1} \\ 2\left(\frac{x-a_{1}}{a_{2}-a_{1}}\right)^{2}, a_{1}<x<b \\ 1-2\left(\frac{x-a_{2}}{a_{2}-a_{1}}\right)^{2}, b<x<a_{2} \\ 1, x \geq a_{2}\end{array}\right.$

$\mu_{\text {bell }}(x)=\left\{\begin{array}{l}\frac{1}{2\left(p-b-a_{1}\right)^{2}}\left(x-a_{1}\right)^{2}, a_{1} \leq x \leq p-b \\ -\frac{1}{2 b^{2}}(x-p)^{2}+1, p-b \leq x \leq p+b \\ \frac{1}{2\left(p+b-a_{2}\right)^{2}}\left(x-a_{2}\right)^{2}, p+b \leq x \leq a_{2} \\ 0, x<a_{1} \text { ili } x>a_{2}\end{array}\right.$ 
$\mu_{1-S}(x)=\left\{\begin{array}{l}1, x \leq a_{1} \\ 1-2\left(\frac{x-a_{1}}{a_{2}-a_{1}}\right)^{2}, a_{1}<x<b \\ 2\left(\frac{x-a_{2}}{a_{2}-a_{1}}\right)^{2}, b<x<a_{2} \\ 0, x \geq a_{2}\end{array}\right.$

Figure 4. S, Bell and Z Membership Functions
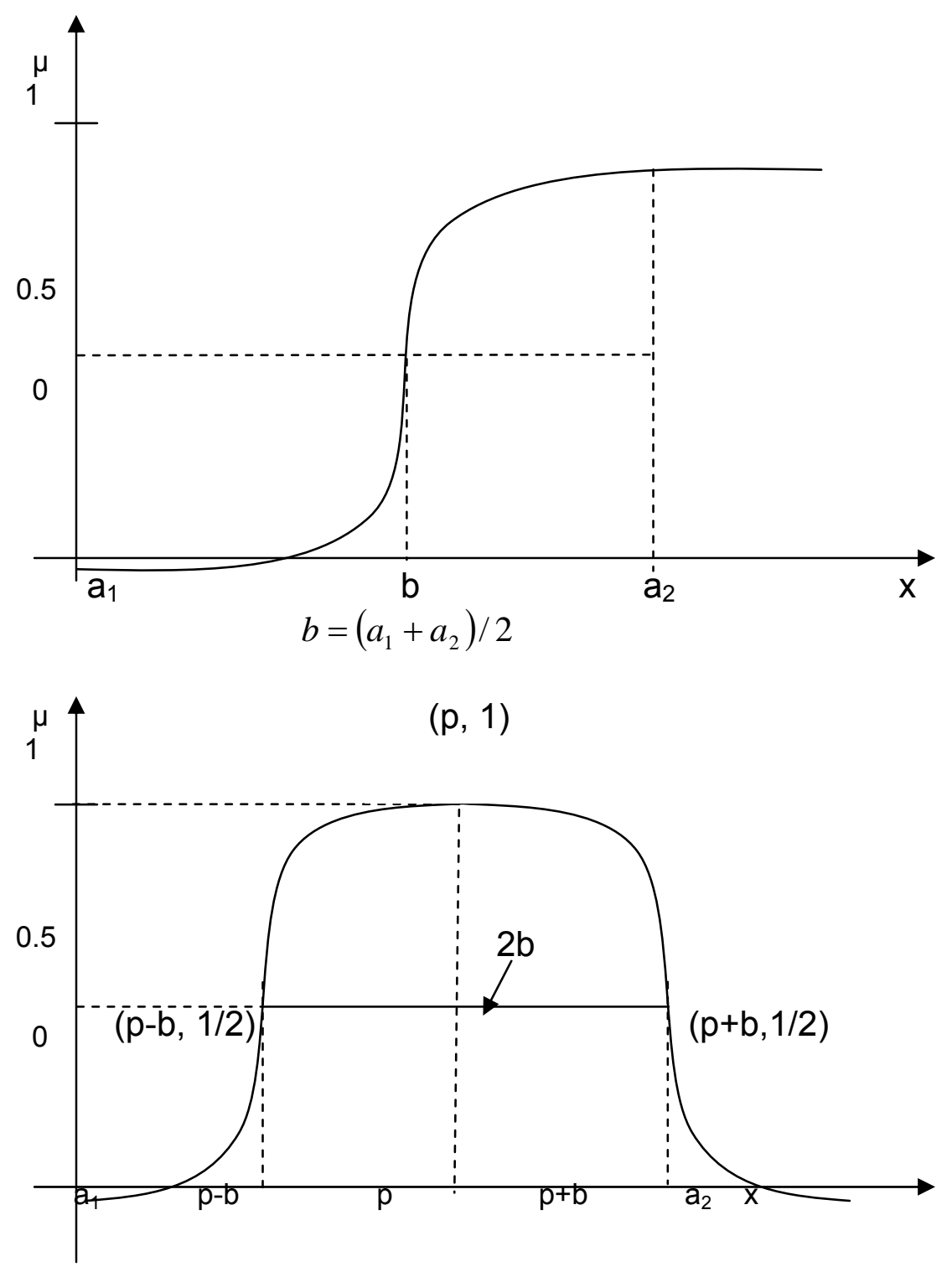


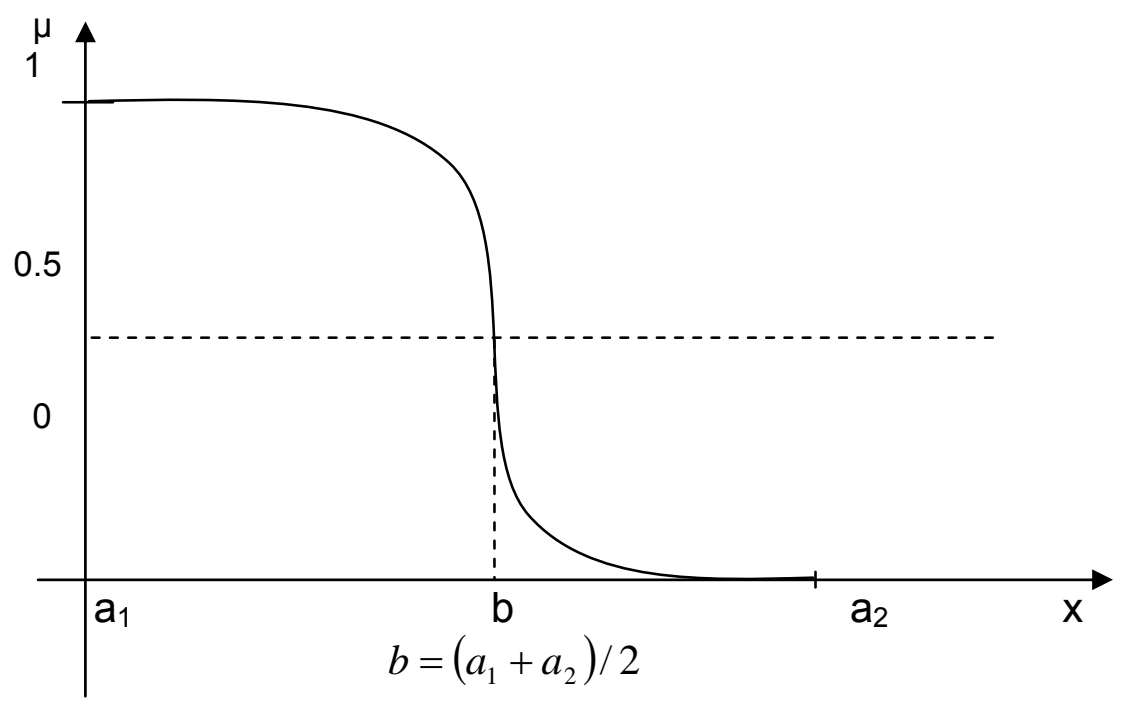

Values of Membership Functions

With fuzzy sets membership function can assume the value of real number from the interval $[0,1]$. The value of membership function to a particular set should be defined for each number of universal set. For example, fuzzy set is marked with $A$. Imagine an universal set containing 6 members, $U=\left\{x_{1}, x_{2}, x_{3}, x_{4}, x_{5}, x_{6}\right\}$ and a fuzzy set $A$ defined with the following values of membership functions:

or

$$
\mu_{\mathrm{A}}\left(\mathrm{x}_{1}\right)=0.15, \mu_{\mathrm{A}}\left(\mathrm{x}_{2}\right)=0.4, \mu_{\mathrm{A}}\left(\mathrm{x}_{3}\right)=0, \mu_{\mathrm{A}}\left(\mathrm{x}_{4}\right)=1, \mu_{\mathrm{A}}\left(\mathrm{x}_{5}\right)=0.2, \mu_{\mathrm{A}}\left(\mathrm{x}_{6}\right)=0.7
$$

$$
A=\left\{x_{1}\left|0.15, x_{2}\right| 0.4, x_{3}\left|0, x_{4}\right| 1, x_{5}\left|0.2, x_{6}\right| 0.7\right\}
$$

For fuzzy sets $A \subseteq U$ and $B \subseteq U$ relations between sets can be determined:

- $A$ is subset of $B$, then and only then, when for $\forall x \in U$ is valid that $\mu_{\mathrm{A}}(\mathrm{x}) \leq \mu_{\mathrm{B}}(\mathrm{x})$.

- $\quad$ A is equal to $B$ only when for $\forall x \in U$ is valid that $\mu_{A}(x)=\mu_{B}(x)$.

- $B$ is complement of $A$ only when for $\forall x \in U$ is valid that $\mu_{B}(x)=1-\mu_{A}(x)$. If a complement is marked with a symbol $\sim$, it is written that $B=\sim A$. So, $\sim(\sim A)=A$ is valid .

- Section $A \cap B$ (fuzzy conjunction) is obtained so that for $\forall x \in U$ membership function value with the section is defined in the following way: $\mu_{\mathrm{A} \cap \mathrm{B}}(\mathrm{x})=\min \left(\mu_{\mathrm{A}}(\mathrm{x}), \mu_{\mathrm{B}}(\mathrm{x})\right)$.

- Union $A \cup B$ (fuzzy disjunction) is obtained so that for $\forall x \in U$ membership function value with the union is defined in the following way: $\mu_{\mathrm{A} \cup \mathrm{B}}(\mathrm{x})=\max \left(\mu_{\mathrm{A}}(\mathrm{x}), \mu_{\mathrm{B}}(\mathrm{x})\right)$.

Linguistic variable «Ability», «Reliability», and «Resulting value» can be modeled by means of sets $A B I, R E L, R E V$ containing certain number of values: 
ability $\triangleq A B I=\left\{A B I_{1}, A B I_{2}, A B I_{3}, A B I_{4}\right\}=\{U N, S A, S U, V S\}$,

reliability $\triangleq R E L=\left\{R E L_{1}, R E L_{2}, R E L_{3}, R E L_{4}\right\}=\{U N, S A, S U, V S\}$,

resulting value $\triangleq R E L=\left\{R E V_{1}, R E V_{2}, R E V_{3}, R E V_{4}\right\}=\{U N, S A, S U, V S\}$,

$U N \underline{\underline{\Delta}}$ unsatisfying, $S A \underline{\underline{\Delta}}$ satisfying, $S U \underline{\underline{\Delta}}$ successful,VS $\underline{\underline{\Delta}}$ very successful.

The terms of linguistic variables ability, reliability, and resulting value have the same triangle membership functions, presented analytically below:

$$
\begin{aligned}
& \mu_{U N}(v)=\left\{\begin{array}{l}
1,1 \leq v \leq 4, \\
\frac{7-v}{3}, 4 \leq v \leq 7,
\end{array}\right. \\
& \mu_{S A}(v)=\left\{\begin{array}{l}
\frac{v-4}{3}, 4 \leq v \leq 7, \\
\frac{10-v}{3}, 7 \leq v \leq 10,
\end{array}\right. \\
& \mu_{S U}(v)=\left\{\begin{array}{l}
\frac{v-7}{3}, 7 \leq v \leq 10, \\
\frac{13-v}{3}, 10 \leq v \leq 13,
\end{array}\right. \\
& \mu_{V S}(v)=\left\{\begin{array}{l}
\frac{v-10}{3}, 10 \leq v \leq 13, \\
1,13 \leq v \leq 16 .
\end{array}\right.
\end{aligned}
$$




\section{Appendix B. Charts, Tables, Formulas, Calculations and Comments}

\section{Evaluation Process}

Model behavior and fuzzy deduction procedure can be described in a few successive steps. First of all, an evaluator must assess the value of input linguistic model variables. Values found are turned into fuzzy sets to show assessment uncertainty - that is called fuzzyfication. Then logic operators are applied to the causative portion of the rule, after that implication procedure from causative to consecutive portion of the rule is made. On the basis of fuzzy rules, the values of output linguistic variables - fuzzy set for each variable - are calculated. Obtained fuzzy sets are aggregated - resulting in a fuzzy set. Finally, on the basis of output fuzzy set, one, precise value of linguistic variable is calculated. It represents the best assessment - defuzzyfication is being implemented.

Fuzzy model presented in this work consists of 3 modules: first two having 2 inputs and 1 output. After calculation, outputs of both modules constitute inputs of the third one, output of which represents the required value.

This way modeling is possible since mathematic prerequisite has been fulfilled - the following axiom has been fulfilled: chosen logic operations are associative; application of addition when working with chosen logic t-norm and t-co-norm operators makes toperation extension for more than two arguments unique.

Figure 5 shows the process flow within the fuzzy evaluation system and its basic parts.

Figure 5. The Model of Fuzzy Evaluation System

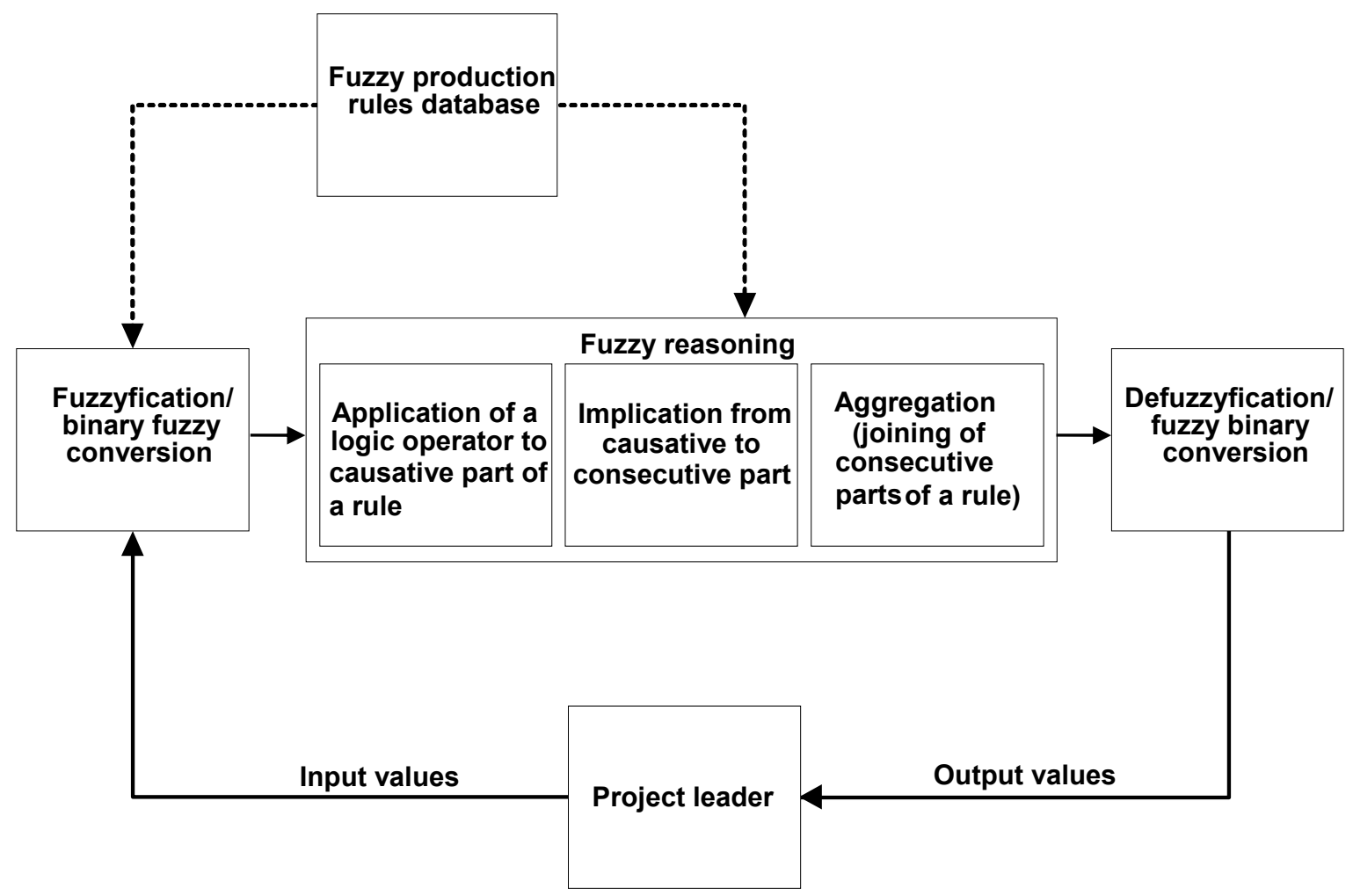


The program manager, based on available information, inputs the values into the input modules of the linguistic variable information system, in which the quantitative values are transformed into quality - the values of fuzzy sets of linguistic variables. They, as input values, present inputs for a module for fuzzy deduction of a production rules database of the «IF-THEN» structure. On the causal parts of the production rules a chosen logical operator is used (in Fuzzy Knowledge Builder ${ }^{\mathrm{TM}}$ this is Zadeh's minimum logic operator). Next an implication is executed from the cause to the consequential part of the rule, and next the consequential parts of the activated rules are joined. The result of the aggregation of the activated rules is a fuzzy set of the output linguistic variable, where the transformation from the quality value into a quantity value is executed. The created output values are used by the program manager as a basis to take appropriate actions, in order to catalyze positive changes and occurrences coming from the actions of individuals that constitute a team. The model is shaped and implemented by using the Fuzzy Knowledge Builder ${ }^{\mathrm{TM}}$ tool programming system, which was used to insert, process and display data. On the basis of phenomena described by linguistic variables the bases of fuzzy rules are made. Figure 6 presents basis of $2^{\text {nd }}$ module fuzzy rules, needed to calculate the value of linguistic variable «Reliability».

\section{Fuzzy Production Rules}

Figure 6. Basis of $2^{\text {nd }}$ Module Fuzzy Rules

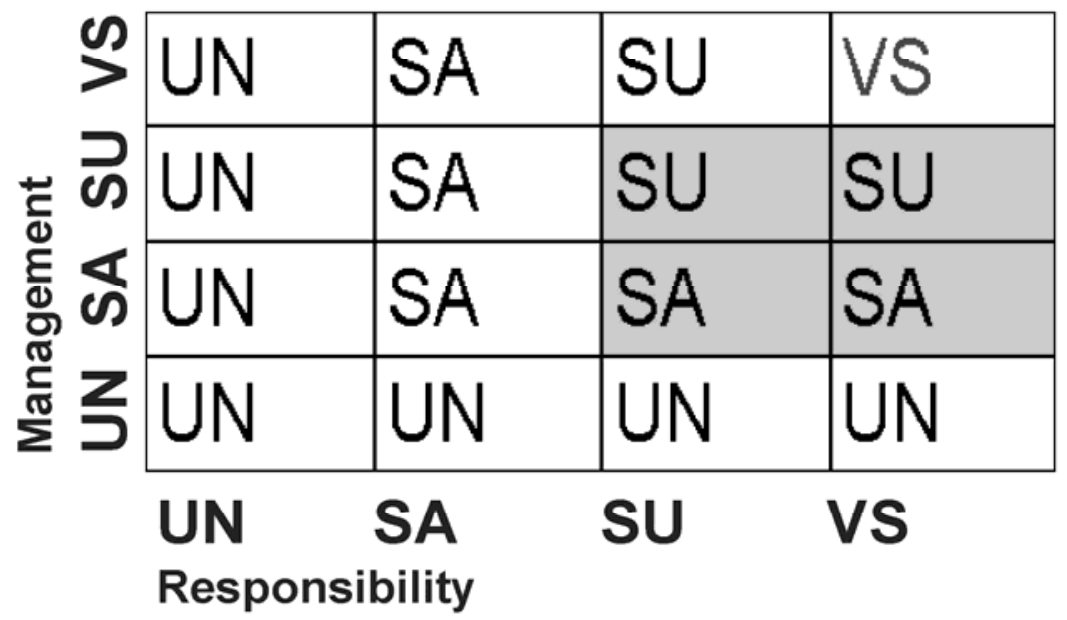


Figure 7 represents calculation rules for output linguistic values $-1^{\text {st }}$ and $3^{\text {rd }}$ module «Ability» and «Resulting value»).

Figure 7. Calculation Rules for Output Linguistic Values

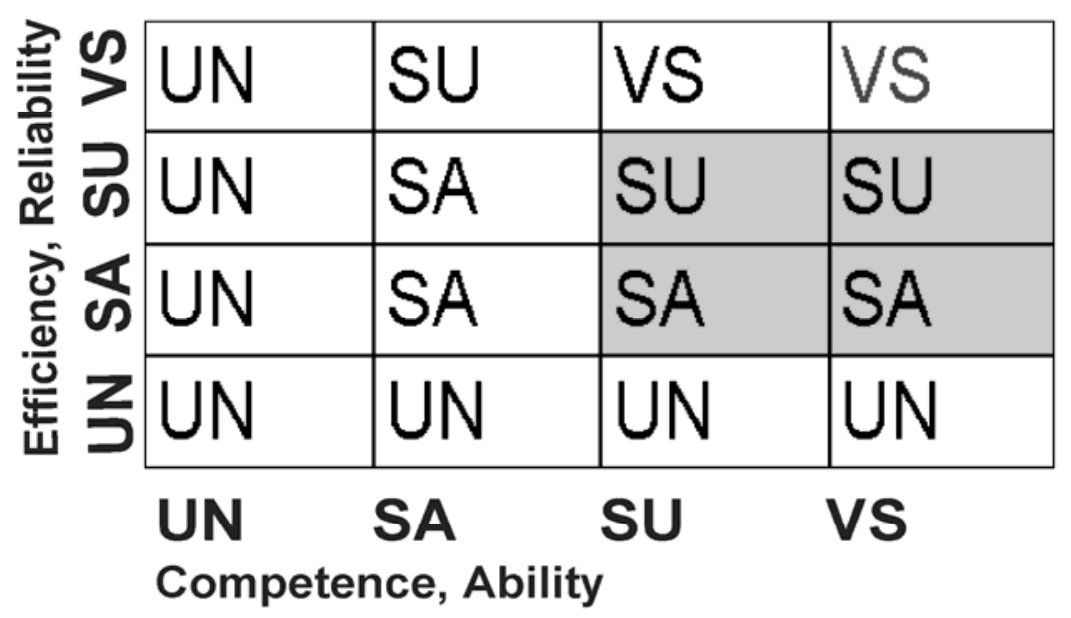

$\mu_{S U}(11)=0.61, \mu_{V S}(11)=0.39, \mu_{S U}(9.1)=0.71, \mu_{S A}(9.1)=0.29$.

Rules activated within third module are:

1. If $A B I$ is $S U$ and REL is $S A$ then $R E V$ is $S A$;

2.If $A B I$ is $S U$ and $R E L$ is $S U$ then $R E V$ is $S U$;

3.If $A B I$ is $V S$ and $R E L$ is $S A$ then $R E V$ is $S A$;

4.If $A B I$ is $V S$ and $R E L$ is $S U$ then $R E V$ is $S U$.

The strength of the rules is calculated as follows:

$$
\begin{aligned}
& \alpha_{11}=\mu_{S U}(11) \wedge \mu_{S A}(9.1)=\min (0.61,0.29)=0.29, \\
& \alpha_{12}=\mu_{S U}(11) \wedge \mu_{S U}(9.1)=\min (0.61,0.71)=0.61, \\
& \alpha_{21}=\mu_{V S}(11) \wedge \mu_{S A}(9.1)=\min (0.39,0.29)=0.29, \\
& \alpha_{22}=\mu_{V S}(11) \wedge \mu_{S U}(9.1)=\min (0.39,0.71)=0.39
\end{aligned}
$$




\section{Values of Evaluation Process}

All input and output processing values produced during evaluation process have been numerically displayed in Table 1 . Finally, following this procedure, the value of output linguistic variable «Resulting value» is reached.

Table 1. Linguistic Variables of a Model With Evaluation Process Values

\begin{tabular}{|cccccc|}
\hline Linguistic variable & Point & $\begin{array}{c}\text { Fuzzy } \\
\text { set }\end{array}$ & $\begin{array}{c}\text { Grade of } \\
\text { membership/ } \\
\text { certainty factor }\end{array}$ & $\begin{array}{c}\text { Alternative } \\
\text { fuzzy set }\end{array}$ & $\begin{array}{c}\text { Alternative } \\
\text { grade of } \\
\text { membership/ } \\
\text { certainty } \\
\text { factor }\end{array}$ \\
\hline Competence & 11 & SU & 0.56 & VS & 0.44 \\
\hline Efficiency & 12 & VS & 0.84 & SU & 0.16 \\
\hline Ability & 11 & SU & 0.61 & VS & 0.39 \\
\hline Responsibility & 12 & VS & 0.91 & SU & 0.09 \\
\hline Management & 9.5 & SU & 0.86 & SA & 0.14 \\
\hline Reliability & 9.1 & SU & 0.71 & SA & 0.29 \\
\hline Resulting value & 8.8 & SU & 0.60 & SA & 0.40 \\
\hline
\end{tabular}

Figure 7 represents 4 rules activated by input values presented in grey colour, calculated output values of afore-going modules. As for observed $3^{\text {rd }}$ module, the values of linguistic variable «Ability» are presented on the horizontal axes, while the values of linguistic variable «Reliability» are presented on the vertical one. To calculate the value of output linguistic variable "Resulting value» in the causative part of the rules being activated, Zadeh's minimum logic operator $T_{M}(x, y)=\min (x, y)$ has been applied, which is one of the basic T-norms. Model can be shaped by tools for fuzzy modeling. Figure 8 and 9 represent input linguistic variables of the $3^{\text {rd }}$ module with values awarded to a team member according to the assessment of the team leader. 


\section{Input Linguistic Variables and Fuzzy Sets}

Figure 8. Input Linguistic Variable «Ability»

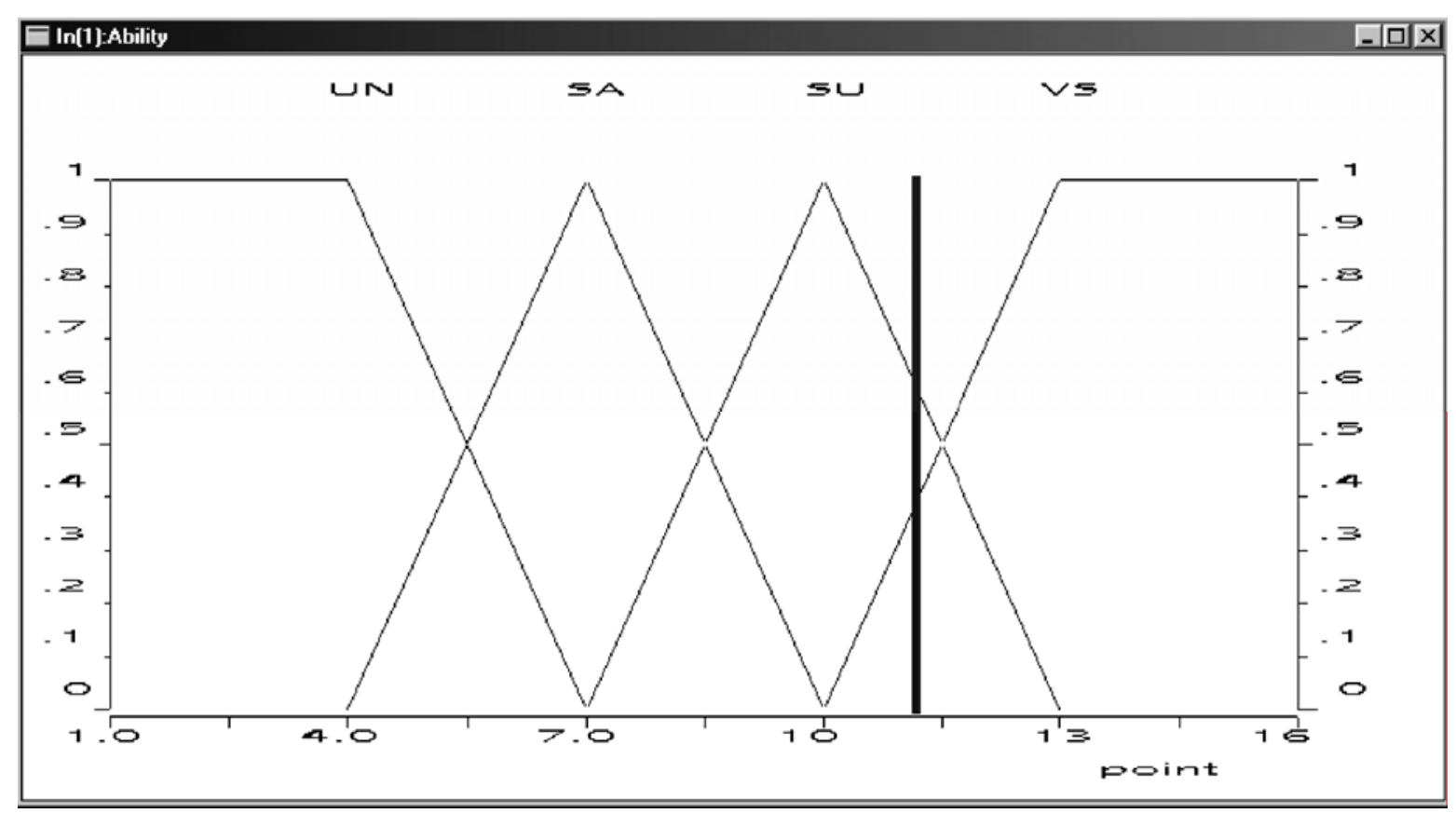

Figure 9. Input Linguistic Variable «Reliability»

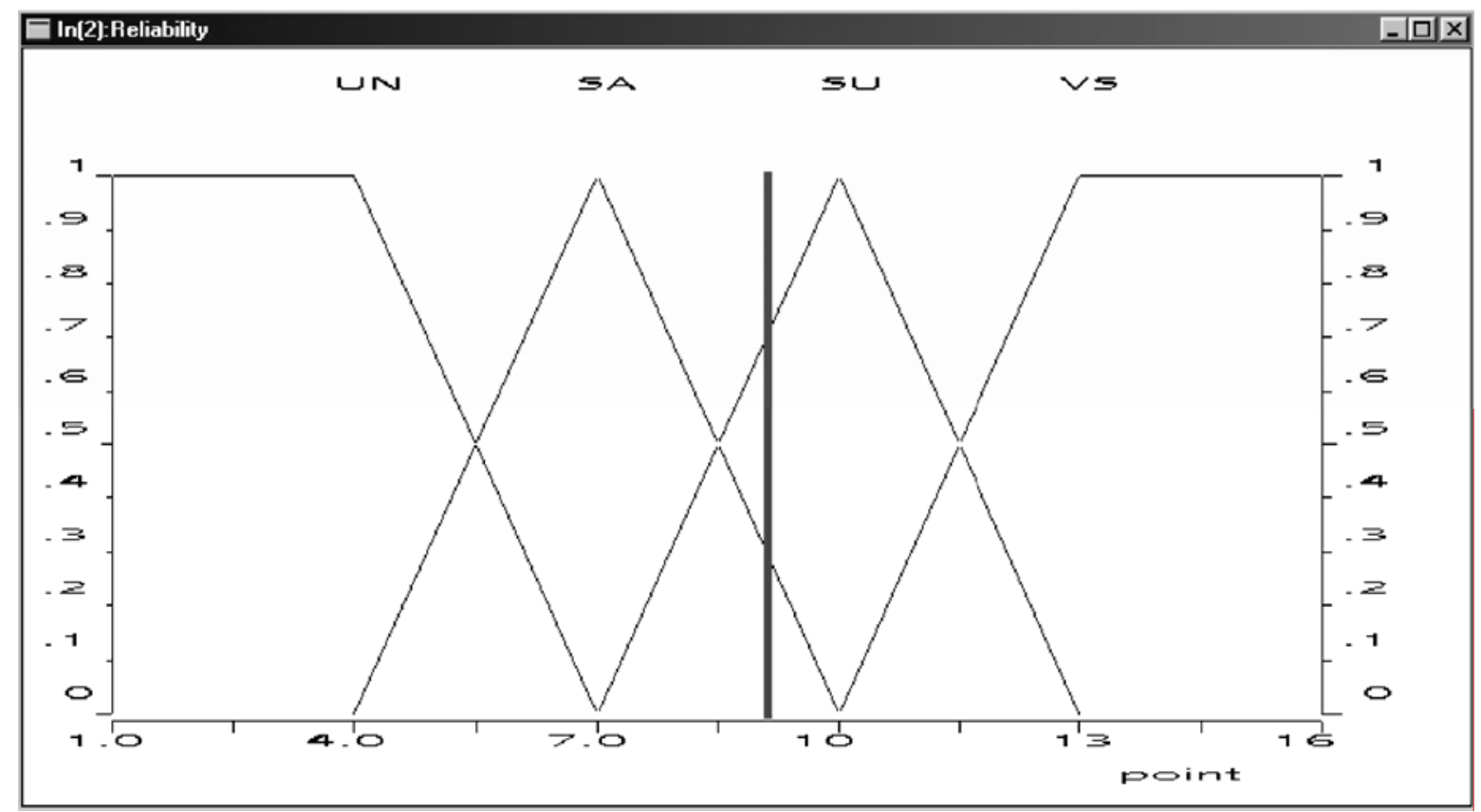

Control output $(\mathrm{CO})$ of each rule is defined by operation conjunction applied to its strength and conclusion: 
CO of rule1: $\alpha_{11} \wedge \mu_{S A}(z)=\min \left(0.29, \mu_{S A}(z)\right)$,

CO of rule $2: \alpha_{12} \wedge \mu_{S U}(z)=\min \left(0.61, \mu_{S U}(z)\right)$,

CO of rule 3: $\alpha_{21} \wedge \mu_{S A}(z)=\min \left(0.29, \mu_{S A}(z)\right)$,

CO of rule $4: \alpha_{22} \wedge \mu_{S U}(z)=\min \left(0.39, \mu_{S U}(z)\right)$.

\section{Output Linguistic Variable - Aggregated Fuzzy Set and Crisp Value}

The aggregated output is:

$\mu_{\text {agg }}(z)=\max \left(\min \left(0.29, \mu_{S A}(z)\right), \min \left(0.61, \mu_{S U}(z)\right)\right)$.

$\mu_{\text {agg }}(z)= \begin{cases}0, & 1 \leq z \leq 4, \\ \frac{z-4}{3}, & 4 \leq v \leq 5, \\ 0.29, & 5 \leq z \leq 8, \\ \frac{z-7}{3}, & 8 \leq v \leq 9, \\ 0.61, & 9 \leq z \leq 11, \\ \frac{13-z}{3}, & 11 \leq v \leq 13 .\end{cases}$

Figure 10 represents output linguistic value, calculation of which brings evaluation procedure to an end.

Figure 10. Output Linguistic Variable «Resulting value» 


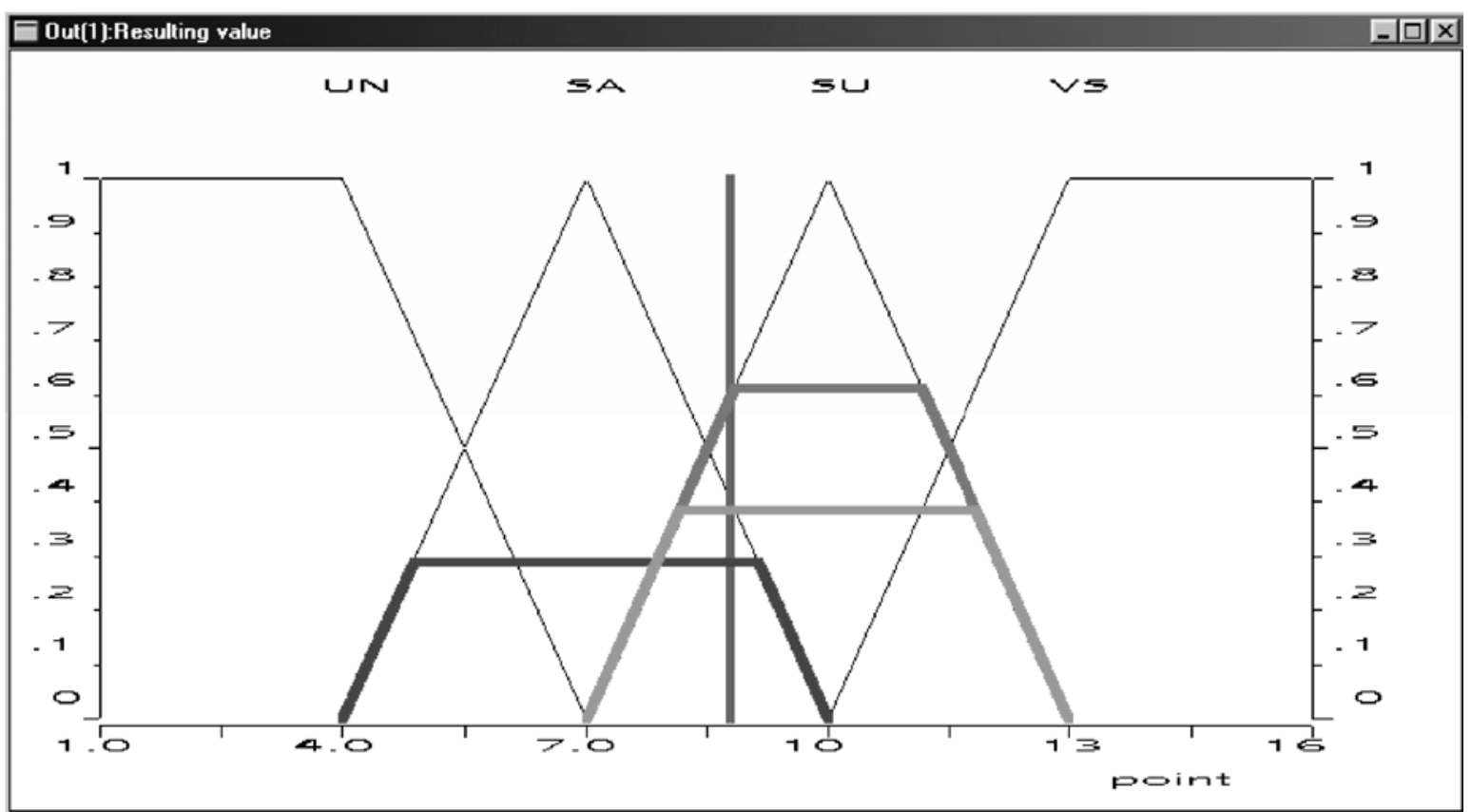

The crisp value of an output linguistic variable is reached by the centre of gravity method. The crisp value $\hat{z}_{c}$ according to this method is the weighted average of the numbers $z_{k}$

$\hat{z}_{c}=\frac{\sum_{k=1}^{q-1} z_{k} \mu_{a g g}\left(z_{k}\right)}{\sum_{k=1}^{q-1} \mu_{a g g}\left(z_{k}\right)}$.

\section{Estimation Surface}

Centre of gravity calculation leads to the crisp value $\hat{z}_{c}=8.8$ which inside fuzzy set "successful» is found on the certainty grade 0.60 while within fuzzy set «satisfying» is on 0.40 . To get better insight into relations between input variables and output variables three-D graph may be used. Figure 11 presents action surface or estimation surface.

Figure 11. Action Surface 


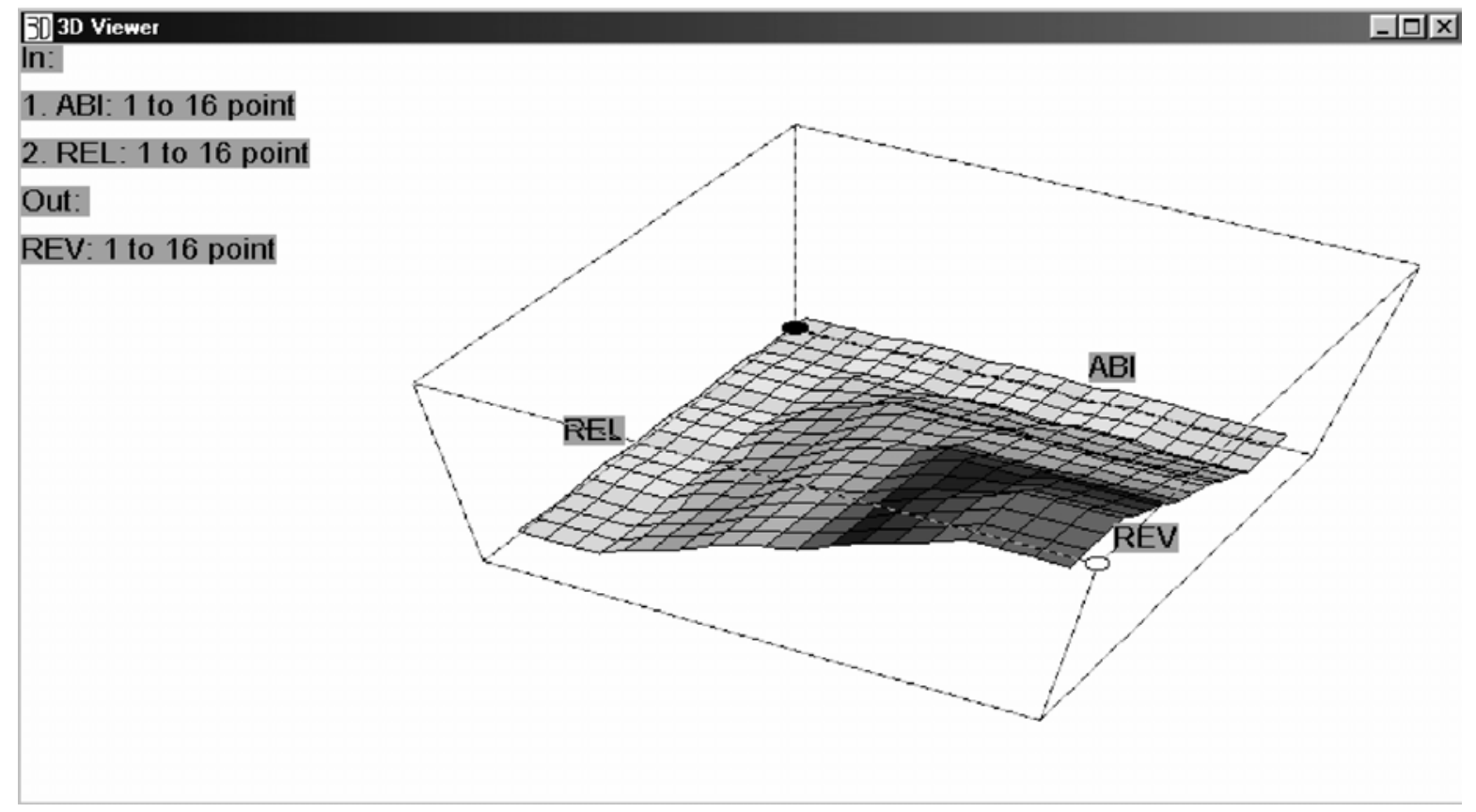

The inputs form the base of the graph, and the output is represented by the height of the graph above each input pair.

\section{References}

Bojadziev, G., \& Bojadziev, M. (1997). Fuzzy Logic for Business, Finance, and Management. Singapore: World Scientific Publishing Co. Pte. Ltd.

Cox, E. (1994). The Fuzzy Systems Handbook, A Practitioner's Guide to Building, Using, and Maintaining Fuzzy Systems. Boston: AP Professional.

Dae-Ho, B., \& Eui-Ho, S. (1994). Human Resource Management Expert Systems Technology. Expert Systems, 11(2), 12-21.

Gabbay, D., \& Guenthner, F. (1994). Handbook of Philosophical Logic Volume III: Alternatives to Classical Logic. Dordrecht: Kluwer Academic Publishers.

Giarratano, H., \& Riley, G. (1998). Expert Systems Principles and Programming. Boston: PWS Publishing Company, An International Thomson Publishing Company Inc.

Gottwald, S. (1996). Many-Valued and Fuzzy Logics. Prague: Eight European Summer School in Logic, Language and Information.

McNeill, F., Thro, M., \& Thro, E. (1994). Fuzzy Logic A Practical Approach. Boston: AP Professional.

Project Management Institute (2000). A Guide to the Project Management Body of Knowledge. North Carolina: PMI Publishing Division. 
Rudas, I. J. (1999). Computational Intelligence in Information/Intelligent Systems, Towards the Generalization of T-Operators: A Distance Based Approach. Proceedings of $10^{\text {th }}$ International Conference «Information and Intelligent Systems - IIS'99», 37-53.

Schneider, M., Kandel, A., Langholz, G., \& Chew, G. (1996). Fuzzy Expert System Tools. Chichester: John Wiley \& Sons. 